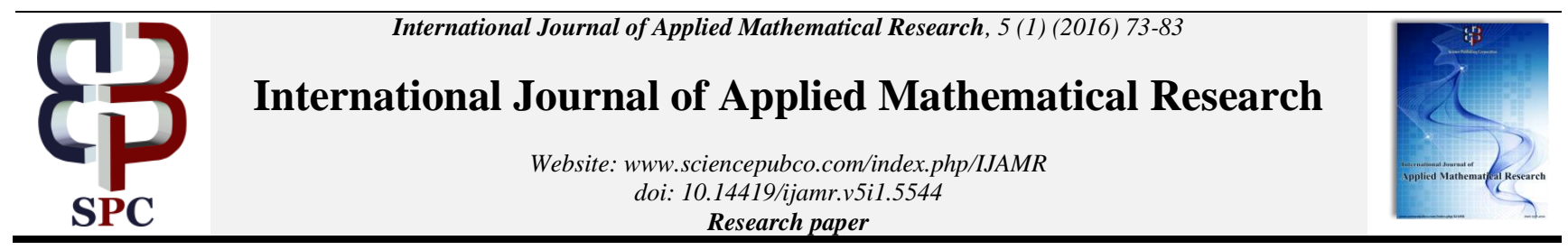

\title{
Modeling metapopulation dynamics of HIV epidemic on a linear lattice with nearest neighbour coupling
}

\author{
Rotich Kiplimo Titus ${ }^{1}$, Lagat Cheruiyot Robert $^{2}$, Pauline Anupi Omulimi ${ }^{3}$, Flomena Jeptanui ${ }^{4}$ \\ ${ }^{I}$ Department of Center for Teacher Education, Moi University, Eldoret, Kenya \\ ${ }^{2}$ Department of Mathematics and Actuarial Science, South Eastern Kenya University, Kitui, Kenya. \\ ${ }^{3}$ Department of Pure and Applied Science, Kisii University, Kisii, Kenya. \\ ${ }^{4}$ Department of Mathematics and Computer Science, University of Eldoret, Eldoret, Kenya \\ *Corresponding author E-mail:tisesko@yahoo.com
}

\begin{abstract}
Many mathematical models for the spread of infectious diseases in a population assume homogeneous mixing, but due to spatial distribution, there exist distinct patches with unique disease dispersion dynamics, especially if between patch mixing due to travel and migration is limited. In this paper, three levels of disease status in a $n$ - patch metapopulation was studied using a simple SIR-HIV epidemic model in a one dimensional nearest neighbour coupling lattice. The basic reproductive ratio $R_{0}(k)$, which is a function of coupling strength $k$, is shown to affect stability characteristics of equilibrium points. The disease free equilibrium (DFE) is globally asymptotically stable irrespective of the value of $k$ but the stability of the endemic equilibrium point (EEP) depends on the coupling strength $k$. It was found that at the critical value of coupling strength $k \geq 0.67$, the subpopulations dynamics are synchronized while for $k \leq 0.3$ the subpopulation dynamics are independent. Patch isolation strategy for the control of HIV dispersion requires a critical coupling strength of $k_{c} \leq 0.15$. This interaction restriction reduces $R_{0}$ to values less than one, and the disease will be eliminated, making isolation effective. Demographic and epidemiological parameters of Vihiga County in Kenya were used in the study.
\end{abstract}

Keywords: Coupling Strength; Isolation; Metapopulation; Reproductive ratio; Synchronization.

\section{Introduction}

The study of infectious diseases has been a major challenge to scientists and epidemiologists, and the use of mathematical models has proven to provide basic information that forms the framework for extended laboratory trials and field experiments. Infectious diseases are either vector borne, airborne, waterborne or contagious. In all these means of transmission, the infected individuals are the immediate neighbours or those who interact with the infectives. Many transmission control strategies are applied at the interface, and their success depends on exact determination of pandemic plume boundaries.

Many epidemic models assume that individuals mix homogeneously implying that all individuals in the population are equally likely to encounter each other making it difficult to determine the width of the spread of an epidemic. In reality, however, many populations are structured in space but interconnected by human travel, migration and by sharing of common facilities. Population may therefore be sub-divided into spatially separated patches also known as the subpopulations, each with its own distinct dynamics. A group of such a distinct subpopulation is known as a metapopulation [10]. Metapopulation is therefore, a fragmented population in which population dynamics occurs at two distinct levels, namely; within patch and between patch. Subpopulation interconnection may be random, all-to-all, one-to-many or nearest neighbour connection topology [21]. In the simplest metapopulation models, individuals are assumed to migrate randomly among patches thus there is no spatial dimension. Such a model is known as spatially implicit model [10]. In this study, we assume nearest neighbour coupling of sub-populations in a one-dimensional lattice. In this case, the interface through which the patches are coupled can be used to delink infected patch from the rest and therefore, control the spread of the disease.

There are different forms of nearest neighbour coupling, which include; coupling on a line; like in chemical reactors systems, coupling on a ring, coupling on a two-dimensional bravais lattice, one-to-all coupling, All-to-all coupling and coupling on a threedimensional bravais lattice. With the definition of coupling as arrangement of subpopulations in a manner that can influence each other, nearest neighbour coupling can represent many physical phenomena, for example, chemical reactors, neural networks, electrical circuits, metallurgy, the spread of an epidemic, just to mention but a few. The last example on the dispersion of infectious diseases in space and time, between subpopulations is considered.

Despite improved sanitation, antibiotics and extensive vaccination programs, infectious diseases continue to be major causes of suffering and mortality. More importantly, infectious disease agents adapt and evolve so that new infectious diseases emerge and existing diseases re-emerge [12]. Diseases that have emerged in recent years include, Hepatitis $\mathrm{C}$ and E, Ebola hemorrhagic fever and Hantavirus. Human Immunodeficiency Virus (HIV) which is the etiological agent of Acquired Immune Deficiency Syndrome (AIDS) emerged in 1981 and has become the leading sexually transmitted disease in the cause of death throughout the world. Drug and antibiotic resistance have become serious issues for diseases such as tuberculosis, malaria and gonorrhoea. Malaria, dengue and yellow fever have re-emerged and are spreading into new regions as climate changes occur. 
Invasion of an infectious agent is considered to be successful if the agent is able to enter into a given patch and spread rapidly within a completely susceptible population. That is, when an initial infected host is able to infect other hosts in the population. When such an invasion occurs, the disease could either go extinct after an initial epidemic or might become endemic in the population without subsequent re-invasion. In homogeneously mixed populations, infectious agents tend to become extinct when the host population size is below a critical community size [10]. However, in metapopulations, the situation become more complex, due to the connectivity of the structure; the infection might become extinct in one patch but simultaneously invade other patches thereby increasing the risk of re-invasion in the future. The between patch dynamics in metapopulation therefore, is important for the persistence of the infectious agent. In addition, to spatial dynamics, demographic and epidemiological processes are important factors that influence persistence of infectious agent in metapopulations. Whether an outbreak of a disease will be an epidemic or endemic depends on the replacement ratio or the basic reproduction tio $R_{0}$. The basic reproduction ratio is the average number of secondary infections produced when one infected individual is introduced into a purely susceptible host population [17]. A disease becomes epidemic if it spreads to a large number of individuals in a given population within a short period of time (usually less than two years) and it is an endemic if it is constantly present to a greater or lesser degree in people of a certain class or certain geographical region [17]. For many deterministic models, an infection can get started in a fully susceptible population if and only if $\mathrm{R}_{0}>$ 1 thus the basic reproduction number is considered as the threshold quantity that determined when an infection invade and persis in a new host population.

The geographic spread of human infectious diseases such as influenza, measles, hemorrhagic fever, severe acute respiratory syndrome (SARS), Ebola, Swine flu and many other infectious diseases are promoted by human travel, which occur on many large scales and are sustained by variety of means of transportation. The dispersal of individuals of a species is one of the key driving forces of various spatiotemporal phenomena that occur on geographical scales. In the light of increasing international travel, the knowledge of dynamical properties of the spread of infectious diseases is of fundamental importance in administering disease control strategies. The understanding of the mechanisms by which the disease spreads and the ability to make predictions about the future course of the epidemic could enable scientists to evaluate inoculation or isolation plans, which may have a significant effect on the control of epidemic spread. Although people interact randomly in an all-to-all manner, the basic building block of interaction pattern is nearest neighbour interaction. It is for this reason that the spread of an epidemic in a one-dimensional linear metapopulation lattice is studied. The effect of coupling strength on synchrony of subpopulation dynamics and on the force of infection is also addressed.

\section{Literature review}

In this section, two main aspects are discussed, namely; epidemiological dynamics in a metapopulation and synchronization of coupled oscillators. The dynamics of an epidemic in a subpopulation will form an oscillator, coupled to other subpopulations in a metapopulation, and their eventual synchrony is discussed.

\subsection{Metapopulation and epidemics}

The scientific study of causes and transmission of diseases in epidemiology is not limited to the origin, development and patterns of disease dispersal. Mathematical models based on the underlying transmission mechanism of a disease helped the scientific community understand the dynamics of disease spread in the community. These models are important because they provide guidelines for application of mitigation strategies through simulation of how changes in the various assumptions and parameter values affect the course of the epidemic. The disease transmission mechanism from the infective to the susceptible is understood for many infectious diseases and the spatial spread of the diseases through a chain of infections is known. However, the transmission interactions in a metapopulation are very complex and are therefore, difficult to understand the large-scale dynamics of the disease spread without the formal structure of the mathematical model.

The study of disease dynamics based on the assumption of homogeneously mixed freely interacting population, where the entire population is considered to be in one group is not realistic. The influence of spatial structure in population on dispersal dynamics of an epidemic is very significant. One way to incorporate structure in epidemiology is to consider metapopulations consisting of well-mixed, coupled patches also known as subpopulations or households. In metapopulation models, the patches are thought to be homogeneously mixed and contain individuals in different states of the disease. There are several choices for the underlying local dynamics, and they can be classified according to the considered phases of the disease (compartments) and the reaction between them. Hufnagel, et al., [9] considered the hierarchy involved in human movements and found that disease spreading is significantly influenced by multilevel movements. Studies based on real human mobility data also provide evidence to support the argument that individual movements occur at different levels [4]. Human mobility tends to be more complex than animal migration or plant dispersal and is not necessarily related to geographic distances [5].

On the metapopulation dynamics, Lloyd and Jansen [13] studied an $\mathrm{n}$-patch model with $\mathrm{k}$ different levels of individuals. Their study concentrated on the linear stability of the spatially homogeneous solutions of the model with population settings in which individuals migrated between patches according to a simple linear term. The dynamic behaviour of the endemic SIR model was decomposed into spatial modes, where the out of phase modes decayed much more rapidly than the in-phase modes for a broad range of coupling strengths. Higher migration rate would mean that an infectious individual would spend shorter time in one patch thus changing patches more frequently. By changing patches frequently, the infectious individual increases the number of contacts with susceptible individuals which increase the probability of the persistence of the infectious agent [11].

A metapopulation model is built based on multilevel movements, including both patch coupling and migration. At the lowest level, where the population movements between the patches are most frequent, the patches will be coupled by the force of infection while patches with fewer frequent movements in between will be linked by migration. An epidemic, in which individuals can distinctly be classified into $\mathrm{n}$ - compartments based on the status of the disease infection, in p Subpopulations can be modelled using a system of np ordinary differential equations. Arino and Van den Driessche [1] studied SEIR model using 4p ordinary differential equations in a metapopulation, where each patch has independent dynamics coupled with the others by means of travel. In their study, they showed that the disease is dying out in each patch if the reproductive ratio $R_{0}<1$ and the disease persists with solutions tending to an endemic equilibrium if $R_{0}>1$. They also observed that if the travel rates of susceptibles and infectives are the same, there exists a unique globally attracting endemic equilibrium. The effect of travelling rates or generally interaction dynamics were also studied by Colizza and Vespignani, [6] using SIR model on metapopulation networks with heterogeneous topology. They derived the basic reaction-diffusion equation describing the metapopulation system at the mechanistic level using degree block variables which allowed them to take into account arbitrary degree distribution of the metapopulation network. Their analytical results showed that along with the usual single population epidemic threshold the metapopulation network exhibits a global threshold for the subpopulation invasion. These results were used to determine the minimum number of individuals travelling among subpopulations in order to have the infection of a macroscopic num- 
ber of subpopulations. This invasion threshold was defined as a function of factors such as the basic reproductive number, the infectious period and the mobility process and it was found to decrease for increasing network heterogeneity.

Intervention strategies like vaccination, isolation or quarantine, treatment, public health educational campaign, use of contraceptives just to mention but a few, will be effective when the disease dynamics is known. Individual strategy may not be successful as observed by Sherry, et al., [19]. In their study, they used the timeseries data for the Ebola virus disease cases to estimate how the rate of exponential rise of new cases had changed over the outbreak and found that in the effective reproduction, number rose when the outbreak spread to densely populated cities, and the enforced quarantine measures were not effective control measures. On the other hand, Rajan, et al., [15] studied control strategies of influenza epidemic to reduce the deaths and established that the optimal vaccination strategy involves concentrating the vaccine on children with the left over vaccine going to the middle aged adults. Their study revealed that given a population of 280 million people, it will be possible to prevent 31 million illnesses more by applying the optimal vaccination strategy when compared with the random mass vaccination. Meanwhile Caitlin, et al. [16], used existing data from Liberia and Sierra Leone to parameterize a mathematical model of Ebola and used the model to forecast the progression of the epidemic, as well as the efficacy of the several interventions, including increased contact tracing, improved infection-control practices, the use of hypothetical pharmaceutical intervention to improve survival in hospitalized patients. Modeling results showed that increased contact tracing and improved infection control or the combination of the two can have a substantial impact on the number of Ebola cases, but the interventions were not sufficient to halt the progress of the epidemic. The hypothetical pharmaceutical intervention while impacting mortality had a smaller impact on the forecasted trajectory of the epidemic.

\subsection{Synchronization of coupled subpopulations}

An Oscillator is a system of model equations, normally differential equations, with a non-constant solution that displays a repetitive behavior and its derivative with respect to time $t$,

If it exists, is non-constant. Examples of oscillators in physical situation include pacemaker.

In the heart, emission of light by fire flies, electric impulses, which propagate along a nerve.

Fiber, periodic outbreak of common diseases, menstrual cycle in women, and periodic behavior of population models just to mention but a few. The dynamics of an infectious disease in one subpopulation is here considered as an oscillator. The periodicity of solution of a system of equations representing infectious disease dynamics is due to complacency and re-infection, especially for diseases, which do not confer immunity [2]. Periodicity of the HIV/AIDS Epidemic in a Mathematical Model that incorporates Complacency was analyzed, and simulations showed that complacency resulting from dependence of HIV transmission on the number of AIDS cases in a community leads to damped periodic oscillations in the number of infective with oscillations more marked at lower rates of progression to AIDS [7]. The implication of these results to public health with respect to monitoring the HIV/AIDS epidemic and widespread use of antiretroviral (ARV) drugs was discussed. In addition, the effect of time delay on the robustness of biological oscillators with respect to varying model parameters showed that time delay destabilize a stable steady state fixed point through Hopf Bifurcations implying oscillating behaviour [2].

We say that synchronization has occurred if two or more oscillators, which previously had different patterns of behavior, begin to behave in the same way and simultaneously such that the knowledge of one can lead to prediction of the behaviour of the other. Analytically, synchronization of coupled oscillators means that there exists a smooth invertible map which carries trajectories on the attractor of one oscillator to the trajectories on the attractor of the other oscillator (see, for instance [8]). Coupled oscillators have been studied extensively for over a long period of time with emphasis in many of the studies being on the existence and stability of limit cycles or equilibrium points. The nature and type of coupling of oscillators yields different topological frames. Considering the infectious disease dynamics in one subpopulation as an oscillator, coupling of different patches in a metapopulation forms a lattice structure which plays significant role in determining the regime of the dynamical behaviour and the dispersal of an epidemic in a metapopulation. The strength of interaction, which in this case is the coupling strength could synchronize populations of interacting species, stabilize them or diversify their group dynamics.

The existence of the synchronization manifold has been the interest of many researchers. Wasike [20] studied diffusive coupling of $\mathrm{n}$-identical subsystems $z_{j} \in R^{N}, j=1,2,3, \ldots, n$ with the dynamics of $z_{j}$ governed by the solutions of the $\mathrm{N}^{\text {th }}$ order ordinary differential equation $\dot{z}_{j}=g_{j}\left(z_{j}\right)$. These systems were coupled with some linear term to obtain $\dot{\mathrm{z}}=\mathrm{A}(\mathrm{k}) \mathrm{z}+\mathrm{f}(\mathrm{z})$, where $\mathrm{f}(\mathrm{z})=$ $\left(\mathrm{g}\left(\mathrm{z}_{1}\right), \mathrm{g}\left(\mathrm{z}_{2}\right), \ldots, \mathrm{g}\left(\mathrm{z}_{\mathrm{n}}\right)\right), \mathrm{k}=\left(\mathrm{k}_{1}, \mathrm{k}_{2}, \ldots, \mathrm{k}_{\mathrm{d}}\right) \in \mathrm{R}^{\mathrm{d}} \quad$ with $\quad$ each $\mathrm{k}_{\mathrm{i}}>0$ a constant positive constant representing the coupling strength and the linear function $\mathrm{A}(\mathrm{k})$ was chosen to be of the form $\mathrm{k} \Delta_{1} \otimes \mathrm{I}_{\mathrm{N}}$, where $\mathrm{k} \geq 0$ is a scalar and $\Delta_{1} \in \mathrm{R}^{\mathrm{n} \times \mathrm{n}}$ is the nearest neighbour coupling configuration matrix and $\mathrm{I}_{\mathrm{N}}$ is the $\mathrm{N}$ dimensional identity matrix. This represents symmetric nearest neighbour diffusive coupling on one dimensional lattice with Neumann boundary conditions. By the invariant manifold theory, he found out the following results: That there exist $\mathrm{k}_{0}$ and a bounded set $\mathrm{U} \in \mathrm{R}^{\mathrm{nN}}$ such that, for each $\mathrm{k}$ with $\mathrm{k}_{\mathrm{j}} \geq \mathrm{k}_{0}, 1 \leq \mathrm{j} \leq$ $d$, the coupled subsystems have a compact global attractor $A_{k} \subset U$ which is uniformly bounded in $\mathrm{k}$, and that there is a critical constant $\mathrm{k}_{0}$ such that the coupled system is synchronized for all $\geq \mathrm{k}_{0}$. Suppose also that the coupled system has a compact global attractor $A_{k}$ for every $k>0$, then we say that the system is synchronized if there exist a bounded diagonal-like, smooth invariant manifold

$\mathrm{M}_{1}:=\left\{\mathrm{z} \in \mathrm{R}^{\mathrm{nd}}: \mathrm{z}_{1}=\mathrm{z}_{2}=\cdots \mathrm{z}_{\mathrm{n}} \neq 0\right\}$

For all $t \geq 0$, if the global attractor $A_{k}$ belongs to the set $M_{1}$. We refer to the set $M_{1}$ as the diagonal or synchronization manifold in $\mathrm{R}^{\text {nd }}$. If $\mathrm{z}$ belongs to the attractor $A_{k}$, it implies that the differences $z_{j}(t)-z_{j+1}(t) \rightarrow 0$ as $t \rightarrow \infty$ for all $1 \leq j \leq n-1$; that is, once the transients have died away, the knowledge of the state of one system allows one to predict the state of the other system.

The synchronization manifold $\mathrm{M}_{1}$ is the most readily observed and of great interest in practical problems. For the system to be synchronized, the diagonal $\mathrm{M}_{1}$ must be invariant under the flow defined by the system equation and locally attracting.

In many applications, one asks for local attraction. In this case, we say that the system is locally synchronized. Local synchronization refers to local attractivity of the set $\mathrm{M}_{1}$ in a small neighbourhood. In this study, synchronization means the subpopulations have attained the same epidemic or endemic status. When endemic status is achieved, it is required that it remains stable and robust. This is a question of stability and persistence. Stability relates to attractivity of the system linearized about the invariant fold $M_{1}$. A system is stable if none of the eigenvalues (for autonomous system) of linearization is positive while Persistence is the ability of the invariant manifold to be insensitive to small perturbations. It refers to the closeness of the invariant manifolds of the perturbed and the unperturbed systems [21].

\section{Model formulation and analysis}

In this section, a mathematical model is formulated using differential equations describing the dynamics of an epidemic in three compartments, namely; Susceptible (S), Infectives (I) and Removed $(\mathrm{R})$. We let $\mathrm{S}(\mathrm{t})$ denote the number of individuals who are 
susceptible to the disease, $\mathrm{I}(\mathrm{t})$ the number of infected individuals, and able to spread the disease by contact with susceptibles and $\mathrm{R}(\mathrm{t})$ the number of individuals were infected at an earlier time and they have been removed either by isolation from the rest of the population, through immunization against infection, through recovery from the disease with full immunity against re-infection, or through adoption of less risk behaviour or by death caused by the disease. It is here assumed that the disease confers a short term immunity, after which the members in this class become susceptible again and can be re-infected, making the model SIRS. It is also assumed that the environment has distinctly isolated patches of subpopulations that can interact in a one dimensional bravais lattice in a line through a nearest neighbour with coupling by means of travel and sharing of common facilities, irrespective of their disease status.

\subsection{Model assumptions, variables, parameters and equations}

In order to formulate the model the following assumptions which describe the relationship between variables and parameters is made.

A1. The entire population is divided into three distinct compartments only with respect to the disease. These are represented by the variables $\mathrm{S}$, I and $\mathrm{R}$, and no other group exists.

A2. The mortality rates are denoted by $\mu_{1}>0$ for Susceptible and $\mu_{2}>0$ for Infective and Removed classes with $\mu_{2}>\mu_{1}$ due to disease related factors leading to accelerated death rate.

A3. Each subpopulation is well mixed and homogeneous. There is no distinction between male and female and they interact freely within the subpopulation. The contacts between individuals on each patch are frequent and hence random mixing applies within the sub-population.

A4. The net migration of individuals between subpopulations is zero.

A5. The recruitment rate into the susceptible class is constant at $\alpha>0$ and additional proportion $\tau$ of the removed individuals is re-admitted into the susceptible class.

A6. Due to natural progression of the disease, an infected individual will move to the recovered class at a rate of $\gamma>0$.

A7. Due to free interaction, on average, a member of the population makes contact sufficient to transmit infection with $\beta \mathrm{N}$ others per unit time, where $\mathrm{N}=\mathrm{S}+\mathrm{I}+\mathrm{R}$ represents total population size (mass action incidence). Accordingly, the probability that an infective comes in contact with a susceptible, who is capable to transmit infection is $\frac{\mathrm{S}}{\mathrm{N}}$, and the number of new infections per unit time for each infective is $(\beta \mathrm{N})\left(\frac{\mathrm{S}}{\mathrm{N}}\right)$, giving the total number of new infections as $\beta$ SI, where $\beta$ is a product $\beta=\mathrm{qc}$ of the number of contacts per unit time $c$ andq: the probability of becoming infected during every contact.

With these assumptions, variables and parameters, a mathematical model representing the dynamics in a single subpopulation is given by the system,

$$
\begin{aligned}
& \frac{\mathrm{d} S}{\mathrm{dt}}=\alpha-\mathrm{k} \beta S \mathrm{I}-\mu_{1} \mathrm{~S} \\
& \frac{\mathrm{dI}}{\mathrm{dt}}=\mathrm{k} \beta S \mathrm{I}-\mu_{2} \mathrm{I}-\gamma \mathrm{I} \\
& \frac{\mathrm{dR}}{\mathrm{dt}}=\gamma \mathrm{I}-\mu_{2} \mathrm{R}
\end{aligned}
$$

\subsection{The n-patch metapopulation model}

Interactions between individuals involve both within-patch interactions and between patch interactions. With the assumption A3, disease dispersal is between batches is largely due to betweenpatch interactions characterized by interaction between individuals from any two neighbouring patches and dominated by frequent movements such as people commuting to and from work, thus interacting with each other in a random manner, but between near- est neighbour batches. A hierarchical system to describe a metapopulation model consisting of 3 levels of movement and having a total of $\mathrm{n}$ patches is formulated and analyzed following the work of Okuonghae and Okuonghae [14] and Bawa et al., [3].

Using system (3.1), we extend the dynamics into a metapopulation with $n$ subpopulations and obtain the model,

$S_{i}^{\prime}(t)=\alpha_{i}-\sum_{j=i-1}^{i+1} k_{i j} \beta_{i j} S_{i} I_{j}-\mu_{1} S_{i}$

$I_{i}^{\prime}(t)=\sum_{j=i-1}^{i+1} k_{i j} \beta_{i j} S_{i} I_{j}-\left(\mu_{2}+\gamma\right) I_{i}$

$R_{i}^{\prime}(t)=\gamma I_{i}-\mu_{2} R_{i}$

For $i=1,2, \ldots, n$ and the constant $k$ is used as a measure of the coupling strength or the force of interaction across and within a subpopulation. In vector form, system (3.2) is represented as;

$X^{\prime}(t)=\Lambda-\beta X^{T}(t) K Y(t)-\mu_{1} X(t)$

$Y^{\prime}(t)=\beta X^{T}(t) K Y(t)-\mu_{2} Y(t)-\gamma Y(t)$

$Z^{\prime}(t)=\gamma Y(t)-\mu_{2} Z(t)$

With

$X=\left(S_{1}, S_{2}, S_{3}, \ldots, S_{n}\right)^{T}, Y=\left(I_{1}, I_{2}, I_{3}, \ldots, I_{n}\right)^{T}, Z=\left(R_{1}, R_{2}, R_{3}, \ldots, R_{n}\right)^{T}$, $\beta=\left(\beta_{i j}\right)$ and $\Lambda=\left(\alpha_{1}, \alpha_{2}, \alpha_{3}, \ldots, \alpha_{n}\right)$ while $K=\left(k_{i j}\right)$ is the tridiagonal coupling matrix given by;

$K=\left(\begin{array}{cccccc}k_{1,1} & k_{1,2} & 0 & \ldots & 0 & 0 \\ k_{2,1} & k_{2,2} & k_{2,3} & \ldots & 0 & 0 \\ 0 & k_{3,2} & k_{3,3} & \ldots & 0 & 0 \\ \vdots & \vdots & \vdots & \ddots & \vdots & \vdots \\ 0 & 0 & 0 & k_{n-1, n-2} & k_{n-1, n-1} & k_{n-1, n} \\ 0 & 0 & 0 & 0 & k_{n, n-1} & k_{n, n}\end{array}\right)$

Here, the subscripts $\left(k_{i j}\right)$ denote the effect from the $i^{\text {th }}$ subpopulation on the $j^{\text {th }}$ subpopulation.

If the subpopulations are equally coupled by a common constant $k$, then matrix (3.4) become

$K=k\left(\begin{array}{cccccc}1 & 1 & 0 & \ldots & 0 & 0 \\ 1 & 1 & 1 & \ldots & 0 & 0 \\ 0 & 1 & 1 & \ldots & 0 & 0 \\ \vdots & \vdots & \vdots & \ddots & \vdots & \vdots \\ 0 & 0 & 0 & 1 & 1 & 1 \\ 0 & 0 & 0 & 0 & 1 & 1\end{array}\right)$

Equally, assuming that the force of infection is equal across the board, $\beta_{i j}=\beta$ then the system (3.2) will reduce to;

$S_{i}^{\prime}(t)=\alpha_{i}-k \beta S_{i} \sum_{j=i-1}^{i+1} I_{j}-\mu S_{i}$

$I_{i}^{\prime}(t)=k \beta S_{i} \sum_{j=i-1}^{i+1} I_{j}-(\mu+\gamma) I_{i}$

$R_{i}^{\prime}(t)=\gamma I_{i}-\mu R_{i}$

Equilibrium points and stability at equilibrium points will be analyzed using model system (3.2) with non-homogeneous coupling strength $K$ given in equation (3.4) together with Neumann boundary conditions.

\subsection{Positivity and boundedness}

Since the model represents the population dynamics of living organisms, positivity and Boundedness is necessary. In order to do this, define a non-negative and ultimately bounded cone $\mathbb{R}=\mathbb{R}_{+0}+$ $\mathbb{R}_{+}$

Positivity

Proposition 3.1. Let the initial conditions of system (3.2) at time $t=0$ be chosen as $S(0)=S_{0} \geq 0, I(0)=I_{0} \geq 0, R(0)=R_{0} \geq 0$. In the following define $\mathbb{R}_{+0}=\{S, I, R \mid S \geq 0, I \geq 0, R \geq 0\}$ and $\mathbb{R}_{+}=\{S, I, R \mid S>0, I>0, R>0\}$. Then the solutions 
$S(t), I(t)$ and $R(t)$ of system (3.1) are positive for all $t>0$. For the model system (3.1), the region $\mathbb{R}$ is positively invariant and all solutions starting in $\mathbb{R}_{+0}$ or $\mathbb{R}_{+}$approach, enter or stay in $\mathbb{R}$.

Proof: Under the given initial conditions, it is easy to prove that the components of solutions of system (3.1), $S(t), I(t)$ and $R(t)$ are positive for $>0$. If not, we assume a contradiction that there exist a first time $t_{0}$ such that $S\left(t_{0}\right)=0, S^{\prime}\left(t_{0}\right) \leq 0$ and $S(t)>$ 0 for $0 \leq t \leq t_{0}$. Consider the first equation of system (3.1), with this assumptions, it follows that the value of the first derivative at $t=t_{0}$ yields

$$
\frac{d S\left(t_{0}\right)}{d t}=\alpha-k \beta S\left(t_{0}\right) I\left(t_{0}\right)-\mu S\left(t_{0}\right)>0
$$

This is a contradiction and thus there exist no such first time and thus $S(t)>0$ for all $t \geq 0$. With $S(t)>0$ for all $t \geq 0$, the second equation of system (3.1) can be integrated by separation of variables as,

$\frac{d I(t)}{d t}=[k \beta S(s)-(\mu+\gamma)] I(t)$

With the solution

$I(t)=I_{0} e^{[k \beta S(s)-(\mu+\gamma)] t}$

Which is positive for all $t \geq 0$. Similarly, the solution to the third equation of system (3.1) can be obtained using integrating factor as, is of the form

$$
R(t)=\frac{\gamma}{\mu_{2}} I(s)+C e^{-\mu_{2} t}
$$

Which is positive for all $t \geq 0$ and $C>0$ arbitrary constant, and $0 \leq s \leq t$.

Boundedness

For boundedness, the sum of the three equations in system (3.1) reduces to $\frac{d N(t)}{d t}=\alpha-\mu N$

With the solution

$N(t)=\frac{\alpha}{\mu}+C e^{-\mu t}$

Taking the limit of solution (3.10) as time $t \rightarrow \infty$, we obtain

$N(\infty)=\frac{\alpha}{\mu}>0$

The solutions in equations (3.7), (3.8), (3.9) and (3.10) show positivity while the limit in equation (3.11) shows Boundedness of the solutions, thus proved.

\subsection{Equilibrium points and stability analysis}

The stability of a system is locally studied near the fixed points, disease equilibrium point (DFE) and endemic equilibrium point (EEP), whose stability depends on the sign of the eigenvalues of a linearized system about the fixed points.

Disease Free Equilibrium (DFE) and its Stability

Using system (3.3), we obtain the disease free equilibrium $E^{0}=$ $\left(S^{0}, I^{0}, R^{0}\right)=\left(\frac{\alpha_{i}}{\mu_{1}}, 0,0\right)$. Stability matrix obtained by linearizing system (3.3) about the fixed point $E^{0}$ is given by

$\left.M\right|_{E^{0}}=\left(\begin{array}{ccc}-\mu_{1} & -\frac{\alpha_{i}}{\mu} \sum_{j=i-1}^{i+1} k_{i j} \beta_{i j} & 0 \\ 0 & \frac{\alpha_{i}}{\mu} \sum_{j=i-1}^{i+1} k_{i j} \beta_{i j}-\left(\mu_{2}+\gamma\right) & 0 \\ 0 & \gamma & -\mu_{2}\end{array}\right)$

Whose eigenvalues are; $\lambda_{1}=-\mu_{1}, \lambda_{2}=\frac{\alpha_{i}}{\mu_{1}} \sum_{j=i-1}^{i+1} k_{i j} \beta_{i j}-\left(\mu_{2}+\right.$ $\gamma)$ and $\lambda_{3}=-\mu_{2}$. Clearly, the first and the last are negative. Stability of system (3.2) at DFE is guaranteed if the second eigenvalue is also negative, that is; $\lambda_{2}=\frac{\alpha_{i}}{\mu_{1}} \sum_{j=i-1}^{i+1} k_{i j} \beta_{i j}-\left(\mu_{2}+\gamma\right)<0 ; i=1,2, \ldots, n$

This condition defines the basic reproductive ratio $R_{0}$ as;

$\frac{1}{\mu_{1}\left(\mu_{2}+\gamma\right)} \sum_{j=i-1}^{i+1} \alpha_{i} k_{i j} \beta_{i j}=\frac{\Lambda \beta K}{\mu_{1}\left(\mu_{2}+\gamma\right)}:=R_{0}$

Where $\Lambda=\left(\alpha_{1}, \alpha_{2}, \ldots, \alpha_{n}\right)$, and $K=\sum_{j=i-1}^{i+1} k_{i j}$.

Endemic Equilibrium Point (EEP) and its Stability

This additional equilibrium point $E^{e}$ of system (3.3) also referred to as has a chronic infection equilibrium denoted by $E^{e}:=$ $\left\{S^{e}, I^{e}, R^{e}\right\}$ is the Endemic equilibrium point where the disease is persistent. This is evaluated from equation (3.3) by solving S, I, R to obtain;

$E^{e}=\left(S^{e}, I^{e}, R^{e}\right)=\left(\frac{\alpha}{\mu_{1} R_{0}}, \frac{\alpha\left(R_{0}-1\right)}{R_{0} k \beta}, \frac{\alpha \gamma\left(R_{0}-1\right)}{R_{0} k \beta \mu_{2}}\right)$,

With $R_{0}$ defined in equation (3.13).

The stability of EEP is determined by the sign of the eigenvalues of linearization matrix evaluated about the fixed point $E^{e}$. This yields the stability matrix

$\left.M\right|_{E^{e}}=$

$\left(\begin{array}{ccc}-\sum_{j=i-1}^{i+1} k_{i j} \beta_{i j} I_{j}^{e}-\mu_{1} & -\sum_{j=i-1}^{i+1} k_{i j} \beta_{i j} S_{i}^{e} & 0 \\ \sum_{j=i-1}^{i+1} k_{i j} \beta_{i j} I_{j}^{e} & \sum_{j=i-1}^{i+1} k_{i j} \beta_{i j} S_{i}^{e}-\left(\mu_{2}+\gamma\right) & 0 \\ 0 & \gamma & -\mu_{2}\end{array}\right)(3.15)$

Whose eigenvalues are $\xi_{1}=-\mu_{2}$ and the other two are obtained from the characteristic equation;

$\xi^{2}-\tau \xi+\delta=0$

Where the coefficients $\tau=$ trace and $\delta=$ determinant are defined as; $\tau=\sum_{j=i-1}^{i+1} k_{i j} \beta_{i j} S_{i}^{e}-\sum_{j=i-1}^{i+1} k_{i j} \beta_{i j} I_{j}^{e}-\mu_{1}-\mu_{2}-\gamma$ and $\delta=\left(\mu_{2}+\gamma\right)\left(\sum_{j=i-1}^{i+1} k_{i j} \beta_{i j} I_{j}^{e}+\mu_{1}\right)-\mu_{1} \sum_{j=i-1}^{i+1} k_{i j} \beta_{i j} S_{i}^{e}$.

EEP is created when $R_{0}>1$ and its stability is determined by signs of the roots of the characteristic equation (3.16). The signs of the characteristic roots can be determined using Routh-Hurwitz stability condition in [18] which requires that the system leading to Equation (3.16) is stable if $\tau<0$ and $\delta>0$. Clearly, $\delta>0$ and thus the system is stable if,

$\tau=\frac{1}{R_{0}}\left(\frac{k \beta \alpha}{\mu_{1}}+1\right)-\left(\alpha+\mu_{1}+\mu_{2}+\gamma\right)<0$.

This gives the critical value $R_{c}$ that guarantees stability as;

$R_{0}>R_{C}=\frac{\alpha k \beta+\mu_{1}}{\mu_{1}\left(\alpha+\mu_{1}+\mu_{2}+\gamma\right)}$

This threshold value guarantees persistence of the disease. For $1 \leq R_{0} \leq R_{c}$ the disease dynamics oscillate. The presence of the coupling constant in the numerator signifies a direct proportion to the critical reproductive ratio $R_{c}$. This implies that there exist a critical coupling strength $k_{c}>0$ such that $R_{c} \leq 1$ which leads to the disease extinction.

\section{Numerical results}

To bring out the analytic solutions in the previous section clear, the analytic results is illustrated with specific numerical example. A complete list of parameters and their estimated values that used for numerical simulations of the model are given in Table 1 .

Data collected from five health facilities are used to represent five distinct metapopulations. The assumption made here is that the subpopulations living around the health centre interact and live in isolation and the cases reported in the next clinic do not contain the same individuals from the other subpopulation. Data collected from five subpopulations in Vihiga County are presented in the 
table below. The Subpopulations $S_{1}, S_{2}, S_{3}, S_{4}$ and $S_{5}$ represents Mbale, Sabatia, Bugina, Kegondi and Vihiga health centres and their immediate environs respectively.

In the simulation of the model (3.2), together with the values of the parameters presented in Table 1 , the following initial values apply. $S(0), I(0), R(0)=(100,0.0001,0)$. Here $I(0)=0.0001$ is used to avoid trivial solution at DFE point. In the simulation, the initial number of infected individuals released into a subpopulation is varied to determine the effects of coupling strength in the velocity of the spread from one subpopulation to the other.

Table 1: Data for the Simulation of the Immune Response to TB Infection Model

\begin{tabular}{|c|c|c|c|c|c|c|}
\hline \multirow[b]{2}{*}{$\begin{array}{l}\text { Parameter } \\
\text { Description }\end{array}$} & \multirow[b]{2}{*}{$\begin{array}{l}\text { Sym } \\
\text { bol }\end{array}$} & \multicolumn{5}{|c|}{ Value(s) of 5 subpopulations } \\
\hline & & Mbale & Sabatia & $\begin{array}{l}\text { Bugi- } \\
\text { na }\end{array}$ & $\begin{array}{l}\text { Kegon } \\
\text { di }\end{array}$ & $\begin{array}{l}\text { Vihi- } \\
\text { ga }\end{array}$ \\
\hline $\begin{array}{l}\text { Naive re- } \\
\text { cruitment } \\
\text { rate in to a } \\
\text { subpopula- } \\
\text { tion }\end{array}$ & $\alpha_{i}$ & 10 & 12 & 8 & 12 & 9 \\
\hline $\begin{array}{l}\text { Force of } \\
\text { infection }\end{array}$ & $\beta_{i j}$ & 0.00427 & 0.0665 & $\begin{array}{l}0.122 \\
3\end{array}$ & 0.0257 & $\begin{array}{l}0.072 \\
3\end{array}$ \\
\hline $\begin{array}{l}\text { Natural } \\
\text { death rate }\end{array}$ & $\mu_{1}$ & 0.008 & 0.008 & 0.008 & 0.008 & 0.008 \\
\hline $\begin{array}{l}\text { Accelerated } \\
\text { death rate } \\
\text { due to in- } \\
\text { fection }\end{array}$ & $\mu_{2}$ & 0.0862 & 0.0977 & $\begin{array}{l}0.106 \\
3\end{array}$ & 0.1039 & $\begin{array}{l}0.078 \\
3\end{array}$ \\
\hline $\begin{array}{l}\text { Re-infection } \\
\text { rate from } \\
\text { removed to } \\
\text { susceptible }\end{array}$ & $\tau$ & 0.1789 & 0.0865 & $\begin{array}{l}0.024 \\
5\end{array}$ & 0.0519 & $\begin{array}{l}0.133 \\
6\end{array}$ \\
\hline $\begin{array}{l}\text { Rate of } \\
\text { progression } \\
\text { from infec- } \\
\text { tive to re- } \\
\text { moved }\end{array}$ & $\gamma$ & 0.0018 & 0.7180 & $\begin{array}{l}0.756 \\
6\end{array}$ & 0.5779 & $\begin{array}{l}0.142 \\
9\end{array}$ \\
\hline $\begin{array}{l}\text { Coupling } \\
\text { Strength } \\
\text { between } \\
\text { Sub- } \\
\text { populations }\end{array}$ & $k_{i j}$ & Variable & the interv & $10 \leq k_{i j}$ & $\leq k_{c}$ (Crit & cal) \\
\hline $\begin{array}{l}\text { Initial Sus- } \\
\text { ceptible } \\
\text { Population } \\
\text { Initial In- }\end{array}$ & $S_{i}$ & 134 & 110 & 90 & 118 & 100 \\
\hline $\begin{array}{l}\text { Initial In- } \\
\text { fected popu- } \\
\text { lation at } \\
\text { DFE }\end{array}$ & $I_{i}$ & 0 & 0 & 0 & 0 & 0 \\
\hline $\begin{array}{l}\text { Initial In- } \\
\text { fected popu- } \\
\text { lation at } \\
\text { EEP }\end{array}$ & $I_{i}$ & 0.001 & 0.001 & 0.001 & 0.001 & 0.001 \\
\hline $\begin{array}{l}\text { Initial Re- } \\
\text { moved } \\
\text { population }\end{array}$ & $R_{i}$ & 0 & 0 & 0 & 0 & 0 \\
\hline
\end{tabular}

The following graphs were generated using MATLAB using Runge-Kutta numerical scheme of order 4-5. We adopt a mesh size of $h=0.01$ in a time interval of $t \in[0,500]$ time units.

The model equations used in programming MATLAB is given by the system below.

$S_{1}{ }^{\prime}(t)=\alpha_{1}-k_{11} \beta_{11} S_{1} I_{1}-k_{12} \beta_{12} S_{1} I_{2}-\mu_{1} S_{1}+\tau R_{1}$

$I_{1}^{\prime}(t)=k_{11} \beta_{11} S_{1} I_{1}+k_{21} \beta_{21} S_{2} I_{1}-\left(\mu_{2}+\gamma\right) I_{1}$

$R_{1}^{\prime}(t)=\gamma I_{1}-\mu_{2} R_{1}-\tau R_{1}$

$S_{2}{ }^{\prime}(t)=\alpha_{2}-k_{21} \beta_{21} S_{2} I_{1}-k_{22} \beta_{22} S_{2} I_{2}-k_{23} \beta_{23} S_{2} I_{3}-\mu_{1} S_{2}+$

$\tau R_{2}$

$I_{2}^{\prime}(t)=k_{12} \beta_{12} S_{1} I_{2}+k_{22} \beta_{22} S_{2} I_{2}+k_{32} \beta_{32} S_{3} I_{2}-\left(\mu_{2}+\gamma\right) I_{2}$

$R_{2}^{\prime}(t)=\gamma I_{2}-\mu_{2} R_{2}-\tau R_{2}$
$S_{3}{ }^{\prime}(t)=\alpha_{3}-k_{32} \beta_{32} S_{3} I_{2}-k_{33} \beta_{33} S_{3} I_{3}-k_{34} \beta_{34} S_{3} I_{4}-\mu_{1} S_{3}+$ $\tau R_{3}$

$I_{3}^{\prime}(t)=k_{23} \beta_{23} S_{2} I_{3}+k_{33} \beta_{33} S_{3} I_{3}+k_{43} \beta_{43} S_{4} I_{3}-\left(\mu_{2}+\gamma\right) I_{3}$

$R_{3}^{\prime}(t)=\gamma I_{3}-\mu_{2} R_{3}-\tau R_{3}$

$S_{4}{ }^{\prime}(t)=\alpha_{4}-k_{43} \beta_{43} S_{4} I_{3}-k_{44} \beta_{44} S_{4} I_{4}-k_{45} \beta_{45} S_{4} I_{5}-\mu_{1} S_{4}+$ $\tau R_{4}$

$I_{4}^{\prime}(t)=k_{34} \beta_{34} S_{3} I_{4}+k_{44} \beta_{44} S_{4} I_{4}+k_{54} \beta_{54} S_{5} I_{4}-\left(\mu_{2}+\gamma\right) I_{4}$

$R_{4}^{\prime}(t)=\gamma I_{4}-\mu_{2} R_{4}-\tau R_{4}$

$S_{5}{ }^{\prime}(t)=\alpha_{5}-k_{54} \beta_{54} S_{5} I_{4}-k_{55} \beta_{55} S_{5} I_{5}-\mu_{1} S_{5}+\tau R_{5}$

$I_{5}^{\prime}(t)=k_{45} \beta_{45} S_{4} I_{5}+k_{55} \beta_{55} S_{5} I_{5}-\left(\mu_{2}+\gamma\right) I_{5}$

$R_{5}^{\prime}(t)=\gamma I_{5}-\mu_{2} R_{5}-\tau R_{5}$

\subsection{Dynamics of susceptible, infectives and recovered in one subpopulation}

The Simulation of the dynamics of the three distinct compartments of model system (3.1) using the data in Table 4.1 is shown in the Fig. 4.1 below. This represents the population dynamics in absence of the disease.

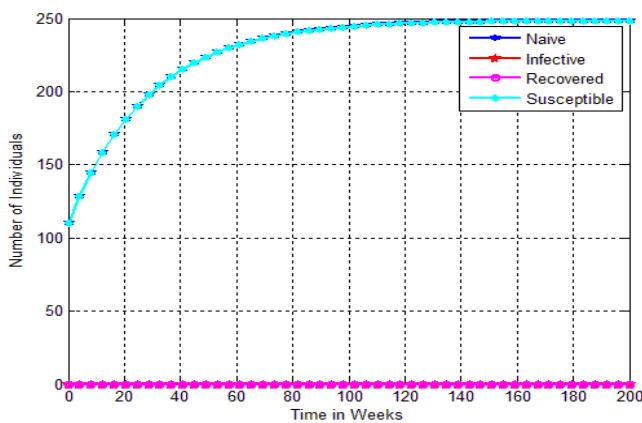

Fig. 4.1: Dynamics of Susceptible, Infectives and Recovered in A Single Subpopulation in Absence of the Disease. Source: Data Simulation.

Clearly, as seen in Fig. 4.1, the dynamics in absence of disease, the population of susceptibles increases steadily, while that of the infectives and recovered remain at zero throughout. Meanwhile, the introduction of one infected individual in the population of purely susceptible population leads to an increase of infectives and recovered as illustrated in Fig. 4.2.

In the study of disease dynamics in a metapopulation, the important parameter is the coupling strength $k_{i j}, i, j=1,2,3,4,5$. This is a measure of how much the subpopulations interact, to allow the transmission of the disease across the boundaries. The boundaries are created by distance, natural barriers like forests, language difference, county administrative boundaries, proximity to health facility, tea plantations, poor road networks, academic institutions and many other factors that create a distinct group and hinder free and frequent interaction between the group and other such groups. 


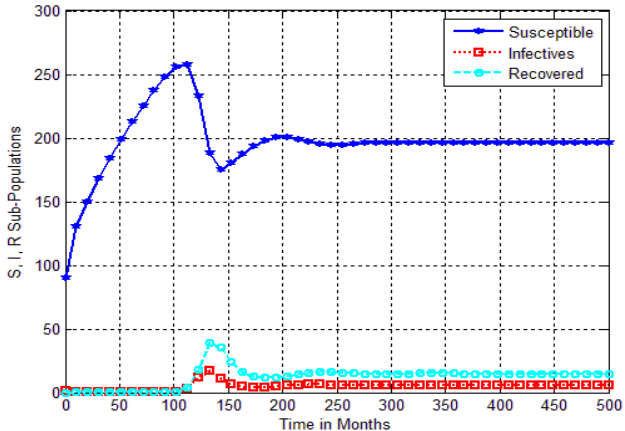

Fig. 4.2: Dynamics of Susceptible, Infectives and Recovered in A Single Subpopulation in Presence of A Disease. Source: Data Simulation.

\subsection{Dynamics of susceptible, infectives and recovered in a metapopulation of five sub-population}

In absence of the disease in the entire metapopulation, the dynamics of the Susceptibles are a reflection of Fig. 4.1, with small differences due to difference in initial populations $S_{i}$. These are illustrated in Fig. 4.3 below. In this simulation, we assume that the force of infection is equal in all subpopulation and the coupling strength $k_{i}=1$ for all groups. This is equivalent to free and unlimited interaction between the subpopulations.

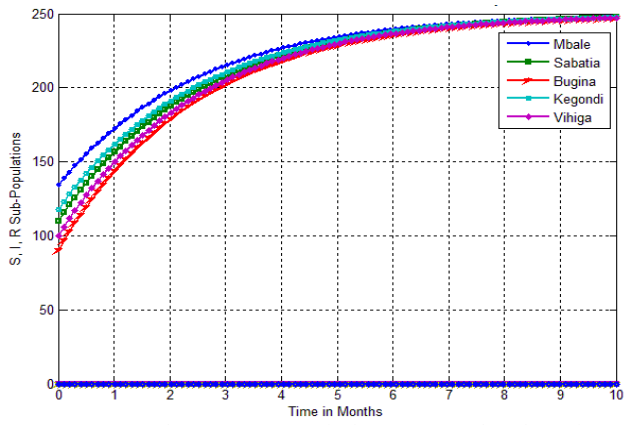

Fig. 4.3: Free Interacting Metapopulation Dynamics in Absence of Disease.

Due to interconnection of the sub-populations, the disease can spread across the boundaries. The force of infection across the sub-population boundaries depends on the strength of interaction between individuals of the sub-populations.

If the coupling strength is strong, introduction of one susceptible in one sub-population will slowly infect the entire metapopulation as shown in Fig. 4.4., but in case of weak coupling strength, the disease will affect one subpopulation where it was introduced. Some of the common barriers that can be used between subpopulations include the use of public health campaign, intensive checks and tests at border entries, use of immunization in a subpopulation, restricting access of facilities outside home region.

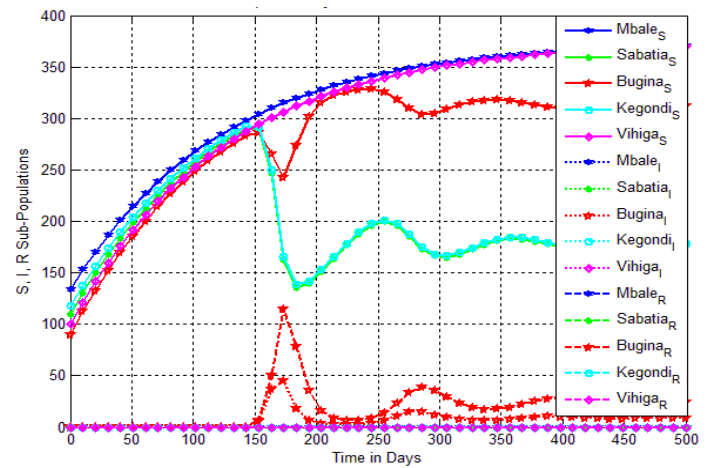

Fig. 4.4: Population Dynamics with Introduction of 1 Infective in Bugina. Notice from the graph the drastic drop in susceptibles of neighbouring sub-populations (Sabatia and Kegondi) and corresponding increase in Infectives and Recoveries of Bugina sub-population.
The infectives are drawn from Susceptibles of the neighbouring subpopulations and once infected; individuals remain in their home subpopulation until they recover. Boundary conditions to restrict the spread of the infection can be implemented to control the spread. Given that $\mathrm{k}_{\mathrm{ij}}$ is the strength of the interaction of subpopulation $i$ and subpopulation $j$, this parameter can be used to measure the level of efforts used to seal the spread of the disease across the boundaries. This parameter will have an inverse effect, so that the more the effort, the lesser the value of $\mathrm{k}_{\mathrm{ij}}$. Total or a complete seal of the boundary is equivalent to $\mathrm{k}_{\mathrm{ij}}=0$. This is a very rare occurrence and $100 \%$ implementation is not easy because people cannot be isolated in the open. This can happen to animals (wildlife or domestic) who can be fenced off from the others. The dynamics in such a case are depicted in Fig. 4.5 below. Only the subpopulation where the infective was introduced will be affected, but all the others, remain steadily growing. Synergetic force of infection is evidenced when one infective is introduced in every subpopulation.

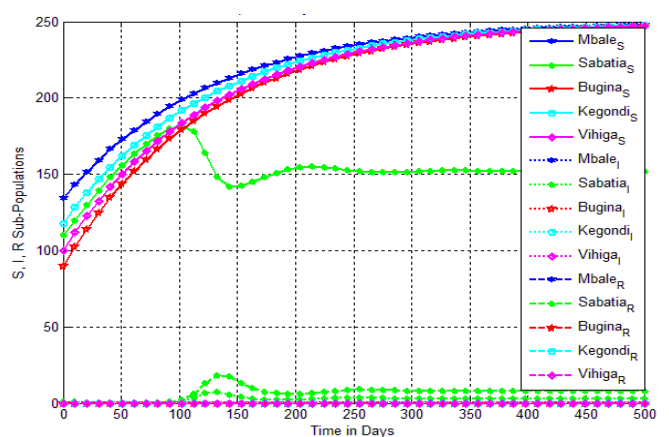

Fig. 4.5: Dynamics of Introducing One Susceptible in an Isolated Metapopulation. Source: Author.

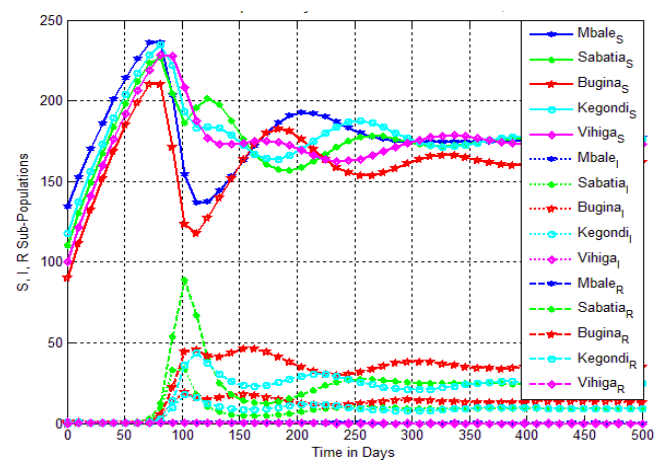

Fig. 4.6: Metapopulation Dynamics when One Infective is introduced in Each Subpopulation. Source: Author.

In absence of any intervention, the population dynamics of people with different disease status is depicted. See Fig. 4.6 above.

\subsection{DFE and EEP steady states and their stability}

There are two steady states as discussed in chapter three, namely Disease Free Equilibrium (DFE) and Endemic Equilibrium Point (EEP). Once any of the steady states is achieved, the system remains stable for any future time. At DFE, the Infectives and Recovered will remain stable at zero while the susceptibles will tend to $S_{0}=\frac{\alpha}{\mu_{1}}$. In our case, this is equal to 250 as simulated and illustrated in Fig. 4.1, 4.3 and 4.5 above. The EEP is experienced when the disease becomes an epidemic. With time, the boundaries between the subpopulations become porous and equal interaction balances the number of infectives in each subpopulation. The concentrations in each group become equal and thus the system achieves a steady state. 


\subsection{Synchronization and coupling strength}

As defined earlier, synchronization is said to have occurred if two or more systems, which previously had different patterns of behaviour begin to behave in the same way and simultaneously such that the knowledge of one can lead to prediction of the behaviour of the other. Denote the five subpopulations as $z_{1}, z_{2}, z_{3}, z_{4}$ and $z_{5}$. Then synchronization means the differences $z_{i}-z_{j}=0, i \neq j$. Using nearest neighbour coupling configuration we obtain the system

$S_{1}{ }^{\prime}(t)=\alpha_{1}-k_{11} \beta_{11} S_{1} I_{1}-k_{12} \beta_{12} S_{1} I_{2}-\mu_{1} S_{1}+\tau R_{1}+k\left(S_{2}-\right.$ $\left.S_{1}\right)$

$I_{1}^{\prime}(t)=k_{11} \beta_{11} S_{1} I_{1}+k_{21} \beta_{21} S_{2} I_{1}-\left(\mu_{2}+\gamma\right) I_{1}+k\left(I_{2}-I_{1}\right)$

$R_{1}^{\prime}(t)=\gamma I_{1}-\mu_{2} R_{1}-\tau R_{1}+k\left(R_{2}-R_{1}\right)$

$S_{2}{ }^{\prime}(t)=\alpha_{2}-k_{21} \beta_{21} S_{2} I_{1}-k_{22} \beta_{22} S_{2} I_{2}-k_{23} \beta_{23} S_{2} I_{3}-\mu_{1} S_{2}+$ $\tau R_{2}+k\left(S_{1}-2 S_{2}+S_{3}\right)$

$I_{2}^{\prime}(t)=k_{12} \beta_{12} S_{1} I_{2}+k_{22} \beta_{22} S_{2} I_{2}+k_{32} \beta_{32} S_{3} I_{2}-\left(\mu_{2}+\gamma\right) I_{2}+$ $k\left(I_{1}-2 I_{2}+I_{3}\right)$

$R_{2}^{\prime}(t)=\gamma I_{2}-\mu_{2} R_{2}-\tau R_{2}+k\left(R_{1}-2 R_{2}+R_{3}\right)$

$S_{3}{ }^{\prime}(t)=\alpha_{3}-k_{32} \beta_{32} S_{3} I_{2}-k_{33} \beta_{33} S_{3} I_{3}-k_{34} \beta_{34} S_{3} I_{4}-\mu_{1} S_{3}+$ $\tau R_{3}+k\left(S_{2}-2 S_{3}+S_{4}\right)$

$I_{3}^{\prime}(t)=k_{23} \beta_{23} S_{2} I_{3}+k_{33} \beta_{33} S_{3} I_{3}+k_{43} \beta_{43} S_{4} I_{3}-\left(\mu_{2}+\gamma\right) I_{3}+$ $k\left(I_{2}-2 I_{3}+I_{4}\right)$

$R_{3}^{\prime}(t)=\gamma I_{3}-\mu_{2} R_{3}-\tau R_{3}+k\left(R_{2}-2 R_{3}+R_{4}\right)$

$S_{4}{ }^{\prime}(t)=\alpha_{4}-k_{43} \beta_{43} S_{4} I_{3}-k_{44} \beta_{44} S_{4} I_{4}-k_{45} \beta_{45} S_{4} I_{5}-\mu_{1} S_{4}+$ $\tau R_{4}+k\left(S_{3}-2 S_{4}+S_{5}\right)$
$I_{4}^{\prime}(t)=k_{34} \beta_{34} S_{3} I_{4}+k_{44} \beta_{44} S_{4} I_{4}+k_{54} \beta_{54} S_{5} I_{4}-\left(\mu_{2}+\gamma\right) I_{4}+$ $k\left(I_{3}-2 I_{4}+I_{5}\right)$

$R_{4}^{\prime}(t)=\gamma I_{4}-\mu_{2} R_{4}-\tau R_{4}+k\left(R_{3}-2 R_{4}+R_{5}\right)$

$S_{4}{ }^{\prime}(t)=\alpha_{5}-k_{54} \beta_{54} S_{5} I_{4}-k_{55} \beta_{55} S_{5} I_{5}-\mu_{1} S_{5}+\tau R_{5}+k\left(S_{4}-\right.$ $\left.S_{5}\right)$

$I_{5}^{\prime}(t)=k_{45} \beta_{45} S_{4} I_{5}+k_{55} \beta_{55} S_{5} I_{5}-\left(\mu_{2}+\gamma\right) I_{5}+k\left(I_{4}-I_{5}\right)$

$R_{5}^{\prime}(t)=\gamma I_{5}-\mu_{2} R_{5}-\tau R_{5}+k\left(R_{4}-R_{5}\right)$

In this model, the parameter $k_{i j}$ denotes the probability of infecting across the boundaries while the parameter $k$ is the coupling strength.

The system above can be written in compact form as;

$\left(\begin{array}{l}z^{\prime}{ }_{1} \\ z^{\prime}{ }_{2} \\ z_{3}^{\prime}{ }_{3} \\ z_{4}^{\prime}{ }_{4} \\ z_{5}^{\prime}\end{array}\right)=k\left(\begin{array}{ccccc}1 & -1 & 0 & 0 & 0 \\ 1 & -2 & 1 & 0 & 0 \\ 0 & 1 & -2 & 1 & 0 \\ 0 & 0 & 1 & -2 & 1 \\ 0 & 0 & 0 & 1 & -1\end{array}\right) \otimes I_{3} \cdot\left(\begin{array}{c}z_{1} \\ z_{2} \\ z_{3} \\ z_{4} \\ z_{5}\end{array}\right)+\left(\begin{array}{c}g\left(z_{1}\right) \\ g\left(z_{2}\right) \\ g\left(z_{3}\right) \\ g\left(z_{4}\right) \\ g\left(z_{5}\right)\end{array}\right)$

Where $z_{i}=\left(S_{i}, I_{i}, R_{i}\right)$ and $g\left(z_{i}\right)$ represent the dynamics of each subpopulation, $I_{3}$ represents the three levels of disease compartments $\mathrm{S}, \mathrm{I}, \mathrm{R}$ and $\otimes$ is the knonecker product.

Equation (4.2) can also be expressed as

$\mathrm{Z}^{\prime}=\mathrm{k} \Delta_{1} \otimes \mathrm{I}_{3} \mathrm{Z}+\mathrm{G}(\mathrm{Z})$

Synchronization is illustrated using numerical simulation of system (4.3) presented in the Fig. 4.7, 4.8 and 4.9 generated by varying the coupling strength. In each of these figures, subplot (a) shows the general dynamics against time, subplot (b) shows oscillatory behaviour of each system, subplot (c) shows the synchronization manifold that is the (diagonal) and subplot (d) shows the differences in the dynamics of the subsystems.

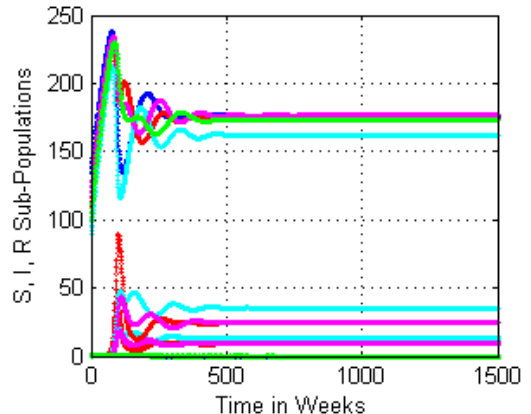

(a) Human Population Dynamics in each Subpopulation

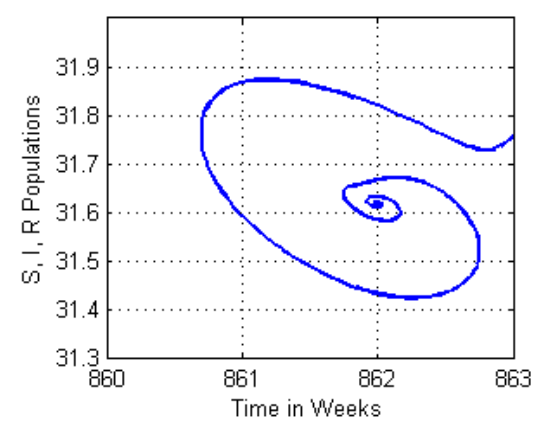

(b) Oscilatory Behaviour of the System

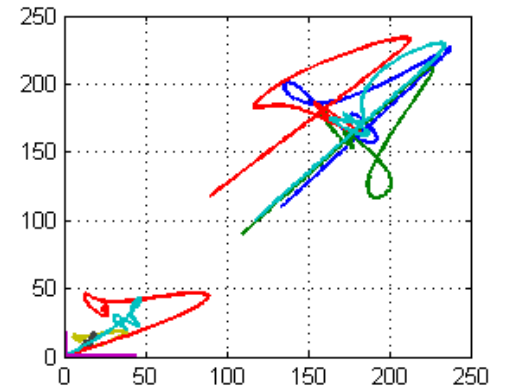

(c) Synchronization Manifold (Diagonal) of the System

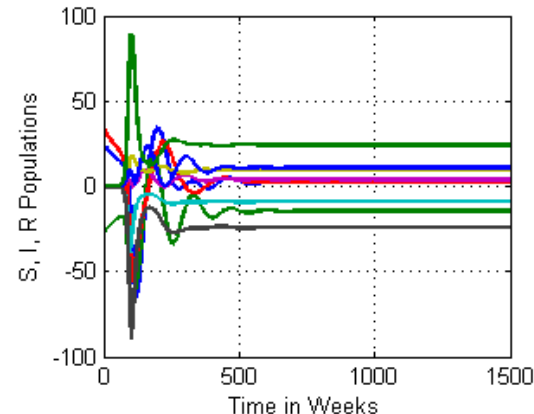

(d) Chaotic Deviations from the Diagonal

Fig. 4.7: Uncoupled System k= 0. Fig. (4.7 (a)) Population Dynamics, (4.7 (b)). Oscillatory Behaviour of Susceptible Against Infectives, (4.7 (c)) Synchronization Manifolds, 4.7 (d)) Chaotic Deviations on Individual Dynamics. Source: Authors Simulation. 
In absence of coupling, the disease dynamics in each subpopulation is independent. This shows a case of isolated patches. Fig. 4.7 above shows the dynamics of uncoupled System with $\mathrm{k}=0$. Notice the chaotic behaviour of the diagonal and the deviations. Also, the first subplot (a) shows distinct lines for each subpopulation. The behaviour of Susceptibles and Infectives is Oscillatory as shown in subplot (b). This implies that each subsystem will converge to its own steady state as time grows. The differences will remain constant.
Due to interaction of individuals between patches, the dynamics of each subpopulation influence each other towards equilibrium. This is simulated by increasing the coupling strength to $\mathrm{k}=0.3$. In Fig. 4.8 , the deviations and the diagonal seem to synchronize, while the individual dynamics converge towards one graph in subplot (a).

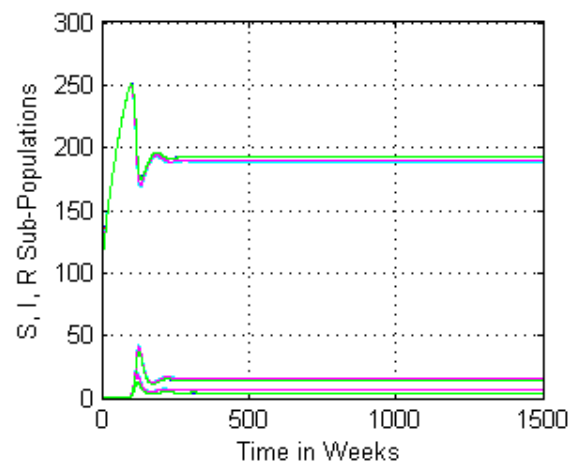

(a) Human Population Dynamics in each Subpopulation

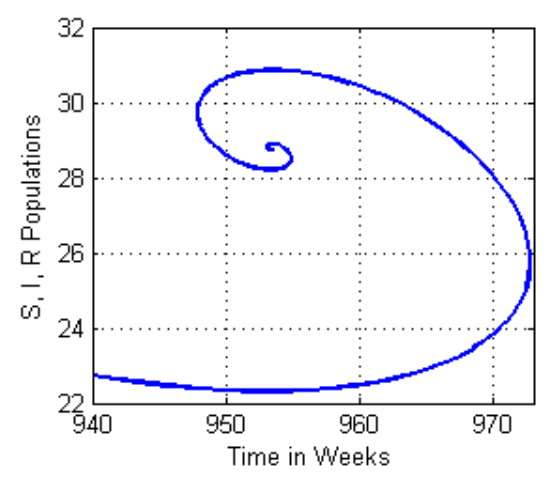

(b) Oscilatory Behaviour of the System

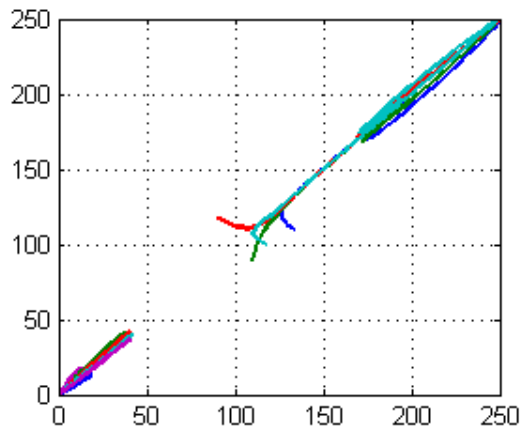

(c) Synchronizations Manifold (Diagonal) of the System

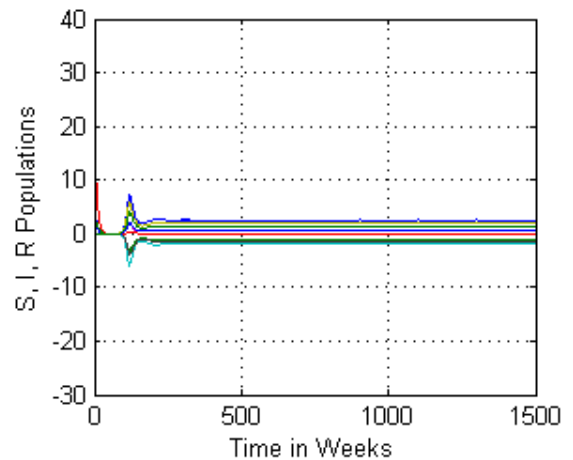

(d) Chaotic Deviations from the Diagonal

Fig. 4.8: Weakly Coupled Systems k = 0.3. Fig. (4.8 (a)) Population Dynamics, (4.8 (b)), Oscillatory Behaviour of Susceptible Against Infectives, (4.8 (c)) Synchronization Manifolds, (4.8 (d)) Chaotic Deviations on Individual Dynamics. Source: Authors Simulation.

Putting in place zero movement restrictions and allowing individuals to mix up freely between patches is equivalent to strongly coupled system at $\mathrm{k}=0.67$. This gives the same results as $\mathrm{k}=1$ or $100 \%$ coupling, where the subpopulations interact like one homogeneous system. Clearly from the graph, all subpopulation graphs coincide to one line and the diagonal together with its transient deviations tends to zero. In this case, the dynamics of one subpopulation is equal to the dynamics of the other subpopulations and the knowledge of one, leads to correct prediction of the other. The population patches are therefore said to be synchronized. The threshold coupling strength necessary to create homogeneity is $\mathrm{k}=0.67$. Distinction of subpopulations can be achieved if interaction is controlled to a level less than $67 \%$. Isolation is successful if interaction is at zero, but anything more than $67 \%$ is not significant. Coupling at $\mathrm{k}=0.67$ is simulated in Fig. 4.9 below.
The desired coupling strength for isolation strategy to be effective requires that the minimum threshold value of $\mathrm{k}_{\mathrm{c}}$ should not be exceeded. This is simulated in Fig. 4.10 and confirms the analytic results. The value of $\mathrm{k}_{\mathrm{c}}$ depends on the force of infection $\beta=\mathrm{qc}$ where $\mathrm{q}$ is the probability that a susceptible can get infected each time when in contact with an infective and $c$ is the number of contacts with infected individuals. Reducing the coupling strength and reduces the number of contacts. The threshold value of coupling for the highest transmission probability $\beta=0.073$ is obtained as $\mathrm{k}_{\mathrm{c}}=0.15$. This means not more than $15 \%$ of the individuals from different patches should be allowed to mix up for isolation strategy to work. 


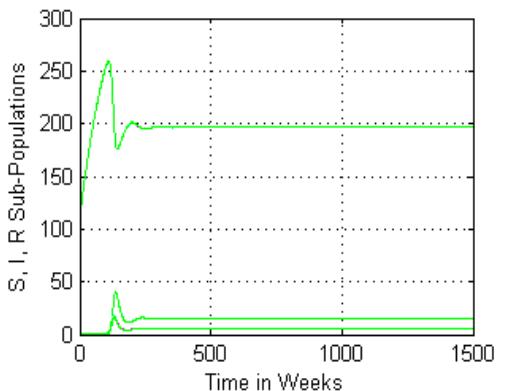

(a) Human Population Dynamics in each Subpopulation

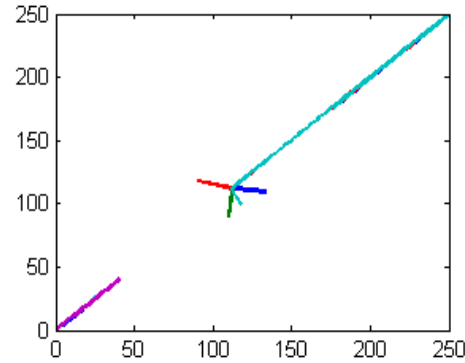

(c) Synchronizations Manifold (Diagonal) of the System

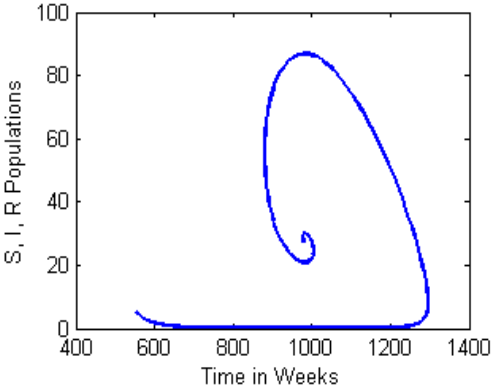

(b) Oscilatory Behaviour of the System

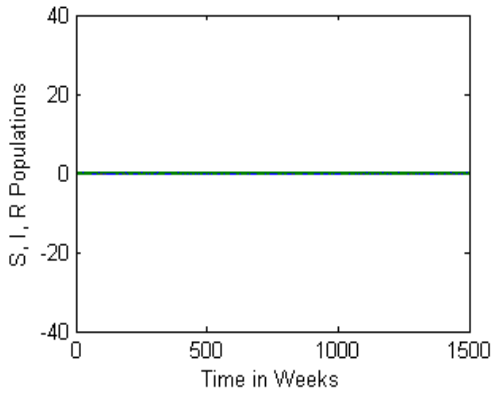

(d) Chaotic Deviations from the Diagonal

Fig. 4.9: Strongly Coupled System $\mathrm{k}=0.67$, (4.9 (a)) Population Dynamics, (4.9 (b)). Oscillatory Behaviour of Susceptible Against Infectives, (4.9 (c)) Synchronization Manifolds, (4.9 (d)) Chaotic Deviations on Individual Dynamics. Source: Authors Simulation.

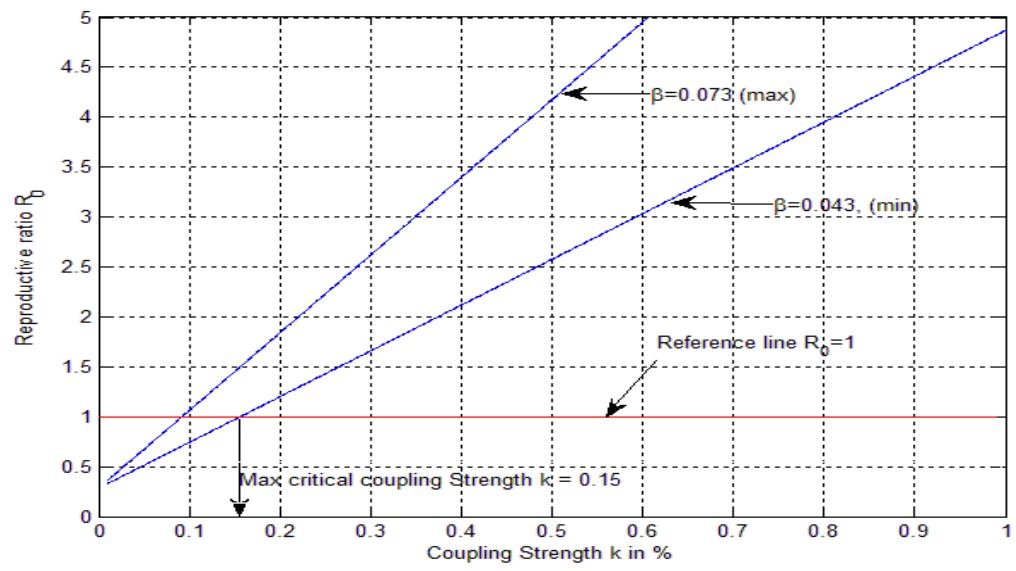

Fig. 4.10: Graph of Coupling Strength $(k)$ Versus Reproductive Ratio $\mathrm{R}_{0}$ Showing the Threshold Maximum Coupling Strength that Guarantees Extinction of the Disease. Source: Author

\section{Summary of the main results, conclusions and recommendations}

The analytic and numerical simulation of the study showed that disease dispersal rates depend on the value of $R_{0}$. This parameter is a function of infection probability, number of contacts and coupling strength. The DFE exists and is stable if $\mathrm{R}_{0}<1$ and for $R_{0}>1$, there exists another equilibrium point EEP where the disease persist. This EEP is stable if $R_{0}>R_{c}$. However, because of the effect of coupling strength on the force of infection, EEP can be eliminated by reducing the coupling strength $\mathrm{k}$ to less than critical value $\mathrm{k}_{\mathrm{c}}=0.15$. When coupling strength is zero $(\mathrm{k}=0)$, there will be as many reproductive ratios as the number of batches but at $\mathrm{k} \geq 0.67$, the batch dynamics are synchronized and the metapopulation behave homogeneously. Since lower values of coupling strength would result in lower $\mathrm{R}_{0}$, it implies that restriction of movement of infected person would help in the control of the disease. We define this as isolation of patches strategy of controlling disease dispersion in a metapopulation.

\section{References}

[1] J. Arino and P. Van Den Driessche, Disease spread in metapopulations, Nonlinear dynamics and evolution equations, 48 (2006), pp. 1-13.

[2] F. Baryarama, L. S. Luboobi and J. Y. Mugisha, Periodicity of the HIV/AIDS epidemic in a mathematical model that incorporates complacency, Am. J. Infect. Dis, 1 (2005), pp. 55-60. http://dx.doi.org/10.3844/ajidsp.2005.55.60.

[3] M. Bawa, S. Abdulrahman, O. Jimoh and N. Adabara, STABILITY ANALYSIS OF THE DISEASE-FREE EQUILIBRIUM STATE FOR LASSA FEVER DISEASE, Journal of Science, Technology, Mathematics and Education (JOSTMED), 9 (2013), pp. 115-123.

[4] D. Brockmann, L. Hufnagel and T. Geisel, The scaling laws of human travel, Nature, 439 (2006), pp. 462-465. http://dx.doi.org/10.1038/nature04292.

[5] D. H. Brown and B. M. Bolker, The effects of disease dispersal and host clustering on the epidemic threshold in plants, Bulletin of mathematical biology, $66 \quad$ (2004), pp. 341-371. http://dx.doi.org/10.1016/j.bulm.2003.08.006.

[6] V. Colizza and A. Vespignani, Epidemic modeling in metapopulation systems with heterogeneous coupling pattern: Theory and simulations, Journal of theoretical biology, 251 (2008), pp. 450-467. http://dx.doi.org/10.1016/j.jtbi.2007.11.028. 
[7] R. V. Culshaw, Mathematical modeling of AIDS progression: limitations, expectations, and future directions, Journal of American Physicians and Surgeons, 11 (2006), pp. 101.

[8] H. Fujisaka and T. Yamada, Stability theory of synchronized motion in coupled-oscillator systems, Progress of Theoretical Physics, 69 (1983), pp. 32-47. http://dx.doi.org/10.1143/PTP.69.32.

[9] L. Hufnagel, D. Brockmann and T. Geisel, Forecast and control of epidemics in a globalized world, Proceedings of the National Academy of Sciences of the United States of America, 101 (2004), pp. 15124-15129. http://dx.doi.org/10.1073/pnas.0308344101.

[10] M. Jesse, P. Ezanno, S. Davis and J. Heesterbeek, A fully coupled, mechanistic model for infectious disease dynamics in a metapopulation: movement and epidemic duration, Journal of theoretical biology, 254 (2008), pp. 331-338. http://dx.doi.org/10.1016/j.jtbi.2008.05.038.

[11] M. Jesse and H. Heesterbeek, Divide and conquer? Persistence of infectious agents in spatial metapopulations of hosts, Journal of theoretical biology, $275 \quad$ (2011), pp. 12-20. http://dx.doi.org/10.1016/j.jtbi.2011.01.032.

[12] R. Levins, T. Awerbuch, U. Brinkmann, I. Eckardt, P. Epstein, N. Makhoul, C. A. De Possas, C. Puccia, A. Spielman and M. E. Wilson, The emergence of new diseases, American Scientist, 82 (1994), pp. 52-60.

[13] A. L. Lloyd and V. A. Jansen, Spatiotemporal dynamics of epidemics: synchrony in metapopulation models, Mathematical biosciences, $\quad 188 \quad$ (2004), $\quad$ pp. http://dx.doi.org/10.1016/j.mbs.2003.09.003.

[14] D. Okuonghae and R. Okuonghae, A mathematical model for Lassa fever, Journal of the Nigerian Association of Mathematical Physics, 10 (2008).

[15] R. Patel, I. M. Longini and M. E. Halloran, Finding optimal vaccination strategies for pandemic influenza using genetic algorithms, Journal of theoretical biology, 234 (2005), pp. 201-212. http://dx.doi.org/10.1016/j.jtbi.2004.11.032.

[16] C. M. Rivers, E. T. Lofgren, M. Marathe, S. Eubank and B. L. Lewis, Modeling the impact of interventions on an epidemic of Ebola in Sierra Leone and Liberia, PLoS currents, 6 (2014). http://dx.doi.org/10.1371/currents.outbreaks.4d41fe5d6c05e9df30d dce33c66d084c

[17] M. Roberts and J. Heesterbeek, Model-consistent estimation of the basic reproduction number from the incidence of an emerging infection, Journal of mathematical biology, 55 (2007), pp. 803-816. http://dx.doi.org/10.1007/s00285-007-0112-8.

[18] A. Thowsen, The Routh-Hurwitz method for stability determination of linear differential-difference systems $\%$, International Journal of Control, 33 (1981), pp. 991-995. http://dx.doi.org/10.1080/00207178108922971.

[19] S. Towers, O. Patterson-Lomba and C. Castillo-Chavez, Temporal variations in the effective reproduction number of the 2014 West Africa Ebola outbreak, PLOS Currents Outbreaks, 6 (2014).

[20] A. A. Wasike, Synchronization and oscillator death in diffusively coupled lattice oscillators, African Journal of Science and Technology, 4 (2003).

[21] A. A. Wasike and K. Rotich, Synchronization and persistence in Diffusively Coupled Lattice Oscillators, (2007) 